\title{
TRP channels in prostate cancer: the good, the bad and the ugly?
}

\author{
Dimitra Gkika ${ }^{1,2}$ and Natalia Prevarskaya ${ }^{1,2}$ \\ During the last decade, transient receptor potential (TRP) channels emerge as key proteins in central mechanisms of the \\ carcinogenesis such as cell proliferation, apoptosis and migration. Initial studies showed that expression profile of some TRP channels, \\ notably TRP melastatin 8 (TRPM8), TRP vanilloid 6 (TRPV6), TRP canonical (TRPC6) and TRPV2, is changing during the development \\ and the progression of prostate cancer towards the hormone-refractory stages. The link between the change in expression levels and the \\ functional role of these channels in prostate cancer is step by step being elucidated. These recent advances are here described and \\ discussed.
}

Asian Journal of Andrology (2011) 13,673-676; doi:10.1038/aja.2011.18; published online 30 May 2011

Keywords: ion channel; lysophospholipids; migration; neuroendocrine; prostate cancer; PSA; transient receptor potential (TRP) channels; TRP channels; TRPM8; TRPV6; TRPV2

\section{TRANSIENT RECEPTOR POTENTIAL (TRP) CHANNELS IN PROSTATE CANCER: A NEW RESEARCH FIELD}

TRP channels constitute a novel area of research in oncology. Malignant transformation of cells is the result of enhanced proliferation, aberrant differentiation and impaired ability to die resulting in abnormal tissue growth, which can eventually turn into uncontrolled expansion and invasion, characteristic of cancer. Such transformation is often accompanied by changes in ion channel expression and, consequently, by abnormal progression of the cellular responses with which they are involved. At present, the expression profile of TRP channels in prostate carcinogenesis has not been yet completed, while the specific functional roles for most of those are only just beginning to be understood.

Since the cloning of the first TRP channel 20 years ago, another approximately 30 TRPs have been identified and classified into six different families: TRP canonical (TRPC), TRP vanilloid (TRPV), TRP melastatin (TRPM), TRP mucolipin, TRP polycystin and TRP ankyrin transmembrane proteins. ${ }^{1}$ Members of the TRP superfamily of channels share a common feature of six transmembrane segments, varying degrees of sequence homology and permeability to cations. Despite these structural similarities, a typical feature of these TRP channels is their ability to be activated by a wide range of external stimuli (including light, sound, chemicals, temperature and touch) as well as of changes in the local environment. As such, they can be envisioned as polymodal molecular sensors of the cell and have profound effects on a variety of physiological and pathological conditions. ${ }^{1-4}$ Sensitivity to polymodal activation suggests that the physiologically relevant stimulus for any given TRP will be governed by the specifics of cellular context (i.e., phosphorylation status, lipid environment, interacting proteins and concentrations of relevant ligands), which dramatically changes during carcinogenesis. TRP channels could therefore play a key role in cancer progression and could characterize the cancer cell phenotype of a tumor. Indeed, this seems to be the case for some of the TRP channels in prostate cancer. The expression levels of members of the TRPC, TRPM and TRPV families are correlated with the emergence and/or progression of certain epithelial cancers. ${ }^{5-8}$ Besides these expression studies, the data of the last couple of years increasingly clarify the role for four TRP members, TRPM8, TRPV6, TRPC6 and TRPV2, making them very promising players since their expression and/or activity mark and regulate specific stages of cancer progression. In this review, we will sketch the emerging profile of TRPM8, TRPV6, TRPC6 and TRPV2 in prostate carcinogenesis and speculate on their potential use in therapeutics. In this regard, the recent advances in the characterisation of the TRPM8 role in cell migration inhibition could reflect a defending, 'Good' character of this channel in prostate cancer invasion. On the other hand, we portrayed as 'Bad' TRPV6 and TRPC6, which show a pro-proliferative role in prostate cancer cells. Finally, in the 'Ugly' stage of the more aggressive hormone refractory cancer, TRPV2 enhances the invasiveness of the prostate cells.

\section{THE 'GOOD': TRPM8 ACTIVATION LEADS TO APOPTOSIS AND PREVENTS PROSTATE CELL MIGRATION}

Trpm 8 was initially cloned as a prostate-specific gene whose expression increased during transformation to prostate cancer. ${ }^{7}$ More precisely, trpm 8 expression increases in both benign prostate hyperplasia and in prostate carcinoma cells, which both present high androgen levels, ${ }^{7}$ while anti-androgen therapy greatly reduces the expression of TRPM8. ${ }^{9}$

${ }^{1}$ Inserm U1003, Equipe labellisée par la Ligue Nationale contre le cancer, Villeneuve d'Ascq, France and ${ }^{2}$ Université des Sciences et Technologies de Lille (USTL), Villeneuve d'Ascq, France

Correspondence: Dr D Gkika (dimitra.gkika@univ-lille1.fr)

Received: 18 February 2011; Revised: 20 March 2011; Accepted: 11 April 2011; Published online: 30 May 2011 
It appears that the androgen dependence of TRPM8 expression is related with the differentiation degree of the prostate epithelial cells. ${ }^{10,11}$ Single-cell RT-PCR and immunohistochemical experiments conducted on primary human prostate cancer cells showed that TRPM8 was mainly expressed in androgen-dependent, apical secretory epithelial cells. Its expression was also downregulated in cells that lost androgen receptor activity. ${ }^{11}$ Furthermore, the epithelial cell phenotype (i.e., fully differentiated secretory apical cells versus nondifferentiated basal cells) and the androgen status (i.e., androgendependent versus hormone refractory cells highly resistant to apoptosis) seem to determine the intracellular TRPM8 localization. Indeed several reports ${ }^{10,12,13}$ indicate that TRPM8 is expressed not only in the plasma membrane $(\mathrm{PM})\left(\right.$ TRPM $\left.8_{\mathrm{PM}}\right)$, as initially anticipated, but also in the endoplasmic reticulum (ER) membrane $\left(\mathrm{TRPM} 8_{\mathrm{ER}}\right)$. Functional TRPM8 $8_{\mathrm{PM}}$ channel is expressed only in highly differentiated prostate epithelial luminal cells, while TRPM8 $8_{\mathrm{ER}}$ localizes in both luminal and basal phenotypes irrespectively of the differentiation status of prostate cells. ${ }^{10}$ The mechanisms, which determine the preferred localization of TRPM8 and the physiological role of this dual expression, are not yet fully clarified.

This dual localization of TRPM8 is of particular interest since it determines the function of the channel and thus, it may be linked to the specific oncogenic status that is critical for carcinogenesis events, such as proliferation, apoptosis and migration. Indeed, activation of TRPM8 $8_{\mathrm{ER}}$ by cold or menthol in LNCaP cells results in the ER store depletion followed by store-operated calcium entry. ${ }^{12}$ Store depletion and store-operated calcium entry were previously shown to be critical in promoting growth arrest and apoptosis of prostate cancer epithelial cells. ${ }^{14,15}$ Indeed, reduced basal filling of intracellular $\mathrm{Ca}^{2+}$ stores is also the hallmark of the apoptosis-resistant cell phenotypes characteristic of advanced prostate cancer. ${ }^{14,16,17}$ Thus, as TRPM $8_{\mathrm{ER}}$ is a molecular entity capable of influencing the filling of ER stores, its activity may be considered a substantial factor in controlling the apoptosis of advanced prostate cancer metastatic cells.

On the other hand, recent data show that TRPM8 $8_{\mathrm{PM}}$ is involved in prostate cancer cell migration. ${ }^{18}$ Activation of the channel either by icilin or the well-known prostate cancer marker, prostate-specific antigen (PSA), reduced the motility of the PC3 prostate cancer cell line. ${ }^{18}$ Regarding the deceleration in cell migration by the newly identified activator of TRPM8, PSA, cell surface biotinylation experiments showed that this is the result of functional TRPM8 channel accumulation on the membrane. ${ }^{18}$ Prostate produces high levels of PSA, also known as the third member of the serine protease kallikrein family, which has been shown to exert anti-angiogenic properties by converting Lys-plasminogen to biologically active angiostatin-like fragments. ${ }^{19}$ Here we show another mechanism through which PSA might slow down prostate tumor growth and progression. PSA activation of the TRPM8 $8_{\mathrm{PM}}$ could thus sustain dormancy of prostatic hyperplasia by reducing its mobility and possible migration. In line with this assumption is the gradual loss of the TRPM $8_{\mathrm{PM}}$ during tumor progression to a late and invasive prostate cancer stage. ${ }^{9}$ Furthermore, another study demonstrates that TRPM8 significantly inhibited the migration in PC3 cells through the inactivation of focal adhesion kinase. ${ }^{20}$ Focal adhesion kinase is a non-receptor protein tyrosine kinase that localizes to cellular focal adhesions or cell contacts within the extracellular matrix and participates in growth factor receptormediated signaling pathways and plays essential roles in cell survival, proliferation, migration and invasion. In addition, overexpression of TRPM8 induced a significant cell cycle arrest in $G_{0} / G_{1}$ stage and facilitated the apoptosis of cells induced by starvation.
Considered together, we can speculate that a shift in the expression and/or activity of TRPM8 may modify the potential for either apoptosis or migration. These studies suggest that TRPM $8_{\mathrm{PM}}$ would play a protective 'Good' role in prostate cancer progression, while it would be edifying to elucidate what is the accountability of the TRPM $8_{\mathrm{ER}}$ in this process. Additional studies will clarify how the balance in between TRPM $8_{\mathrm{PM}}$ and TRPM8 $8_{\mathrm{ER}}$ affects carcinogenesis. Furthermore, there is an urgent need of establishing appropriate experimental in vivo models in order to validate pro-apoptotic/anti-invasive role of TRPM8 and assess whether overexpression or specific activation of either isoforms should be considered and may offer novel approaches to treat prostate cancer depending on the stage and androgen sensitivity of the targeted tumor.

\section{THE 'BAD': TRPV6 AND TRPC6 LEAD TO PROLIFERATION OF PROSTATE CANCER CELLS}

TRPV6 is proposed as a prognostic marker of prostate cancer progression, since its expression strongly increases in high-grade tumors with a Gleason score of $\geqslant 7$, while in normal and benign prostate tissues, it is virtually undetectable. ${ }^{21}$ Using the model system of androgendependent human prostate cancer LNCaP cell line, a recent study showed that TRPV6 is directly involved in the control of proliferation, as siRNA-mediated TRPV6 silencing slowed down the proliferation rate, decreased the accumulation of LNCaP cells in the S phase of the cell cycle and lowered the expression of the proliferating cell nuclear antigen. ${ }^{22}$ The proproliferative role of TRPV6 consists in supporting the basal $\mathrm{Ca}^{2+}$ entry required for the activation of $\mathrm{Ca}^{2+} /$ calmodulin/ calcineurin-dependent transcription factor nuclear factor of activated T cell (NFAT), ${ }^{22}$ whose transcriptional activity alters the expression of cell cycle regulators.

Note worthily, it was demonstrated that the activation of the same $\mathrm{Ca}^{2+} /$ calmodulin/calcineurin/NFAT pathway leads to proliferation in the primary human prostate cancer epithelial cells by $\mathrm{Ca}^{2+}$ entry via another TRP family member-TRPC6. ${ }^{6}$ However, in this case, agonist-mediated stimulation of $\alpha 1$-adrenoceptor is required to generate the lipid messenger diacylglycerol, by recruiting a phospholipase Ccatalyzed phospholipid breakdown pathway, which in turn directly gates TRPC6 channels. ${ }^{6}$ Furthermore, the $\alpha 1$-adrenoceptor must be expressed as a full-length isoform in order to initiate the calcium signal since the lack of the long receptor isoforms hinders $\mathrm{Ca}^{2+}$ entry. Indeed, in DU145 cells, treatment with phenylephrine provokes a recruitment of TRPC6-containing vesicles toward the plasma membrane as well as a rearrangement of the submembranous cytoskeleton, a rearrangement of the receptor containing microdomains along with a modification of microdomain lipidic composition, as seen by an increase in ceramides, cholesterol, sphingomyelin and phosphatidylserin leading to a shift toward lighter densities of the rafts and a modification of the membrane fluidity. ${ }^{23}$

Taken together, it would seem that the TRPV6 channel is involved in the control of basal proliferation rate of prostate cancer cells, while TRPC6 supports the direct mitogenic effect of catecholamines via their release by sympathetic nerves. We could therefore consider TRPV6 and TRPC6 as the 'Bad guys', the inhibition of which could lead to the control of tumor progression in prostate cancers.

\section{THE 'UGLY': TRPV2 CHANNEL ENHANCES PROSTATE CANCER CELL MIGRATION AND INVASION}

TRPV2 is expressed in the more aggressive androgen-resistant prostate cancer cells and its expression is increased by treatments inducing neuroendocrine differentiation. ${ }^{24}$ Indeed, as mentioned above, 
neuroendocrine differentiation has been shown to be associated with the androgen-independent stage of prostate cancer. ${ }^{25}$ This neuroendocrine phenotype of prostate cancer cells is dependent on the presence of TRPV2, since its inhibition by siRNA downregulates neuron-specific enolase, a neuroendocrine marker. ${ }^{24}$

Precisely, TRPV2 is expressed in several metastatic androgen-resistant prostate cancer cell lines PC-3, DU-145 and LNCaP C4-2 and most importantly trpv2 transcript levels were 12 times higher in patients with metastatic cancer (stage M1) compared with primary solid tumors (stages T2a and T2b). Silencing of this channel drastically reduces the migration of prostate cancer cells, whereas its overexpression increases their migration. Concerning the mechanistic role of TRPV2 in invasion, TRPV2 would maintain an elevated level of cytosolic $\mathrm{Ca}^{2+}$ in cancer cells derived from castration-resistant tumors due to its constitutive channel activity. ${ }^{24}$ The activity of this channel would affect prostate cell migration by directly regulating the invasion markers matrix metalloproteinase MMP2, MMP9 and cathepsin B used by the cancer cell to invade.

Physiological regulation of the TRPV2 channel was so far been poorly described. It was, however, shown that this channel can be activated when plasma membrane targeting is stimulated by growth factors (insulin-like growth factor-1), phosphatidylinositol-3 kinase pathway and osmotic stimuli. ${ }^{26}$ Interestingly, the common endogenous lysophospholipids, lysophosphatidylcholine and lysophosphatidylinositol stimulate $\mathrm{Ca}^{2+}$ influx and increase the migration potential in prostate cancer PC3 cells. On the other hand, TRPV2 silencing with shRNA abolishes the stimulatory effect of lysophospholipids on both $\mathrm{Ca}^{2+}$ entry and migration, pointing to a role played by TRPV2 channels in cancer cell migration. ${ }^{24,27}$ These findings are more important in light of the fact that lysophospholipids are significant actors in tumor development, since they stimulate angiogenesis, growth, survival and migration of prostate malignant cells. $^{28-30}$

Thus, TRPV2 would be the 'Ugly guy' that participates in the setting of advanced prostate cancer by enhancing cell migration for more aggressive prostate cells and could constitute a prospective prognostic marker and a potential therapeutic target for future interventions to increase the life expectancy of the patients.

\section{CONCLUSION AND PERSPECTIVES}

Accumulating evidence tends to demonstrate that the development of some cancers could also involve TRP channel changes in expression and/or activity. Indeed concerning prostate cancer, TRPM8, TRPV6, TRPC6 and TRPV2 play distinct roles in different stages of the tumor genesis and/or progression and appear as being instrumental in fundamental tumorigenic processes such as cell growth, proliferation, apoptosis, migration and invasion (summarized in Figure 1). However, it should be noted that it is unclear at present whether these TRP aberrations are a cause or a consequence of tumor genesis, while there is a definite need for more appropriate prostate epithelial cell models, such as primary cultures of prostate epithelial cells.

The potential clinical relevance of these recent advances in the TRP channel field is two-folded: TRP channels may be useful biomarkers for cancer prognosis and may constitute anticancer targets in therapeutics. Concerning their relevance as biomarkers, it has already been suggested that TRPM8 and TRPV6 expression in prostate cancer may constitute prognostic and/or diagnostic markers for prostate cancer, ${ }^{7,8,31}$ The additional use of TRPC6 and TRPV2 as biomarkers and the use of a simultaneous screening for all the aforementioned TRP channels (TRPM8, TRPV6, TRPC6 and TRPV2) could constitute a prognostic/diagnostic profile that would

\section{TRPM8}

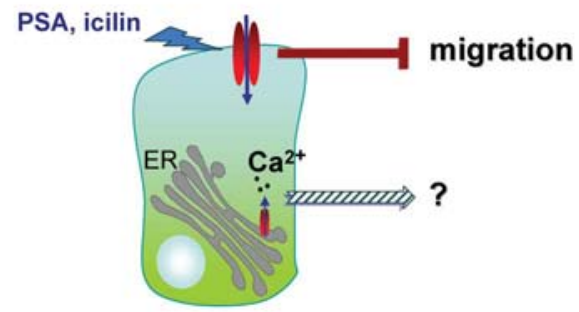

TRPV6

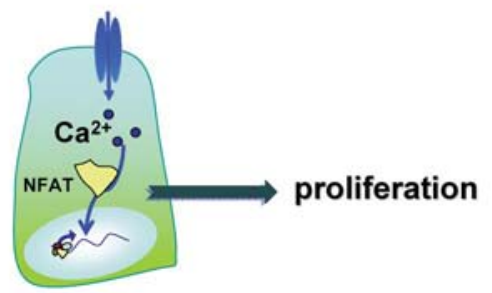

TRPV2

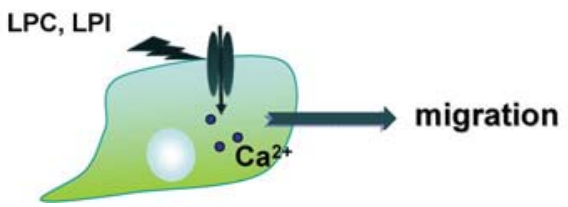

Figure 1 Role of TRP channels in prostate carcinogenesis. Activation of the plasma membrane TRPM8, the 'Good', by icilin or the PSA inhibits prostate cell migration, while the role of this channel on the ER remains to be defined (upper panel). On the other hand, increased basal $\mathrm{Ca}^{2+}$ entry through TRPV6, the 'Bad', in cancer prostate cells, enhances cell proliferation through the activation of $\mathrm{Ca}^{2+} /$ calmodulin/calcineurin-dependent transcription factor NFAT (middle panel). Finally, activation of TRPV2, the 'Ugly', by lysophospholipids such as LPC and LPI, stimulates $\mathrm{Ca}^{2+}$ influx and increase the migration potential in prostate cancer cells (lower panel). ER, endoplasmic reticulum; LPC, lysophosphatidylcholine; LPI, lysophosphatidylinositol; NFAT, nuclear factor of activated T cell; PSA, prostate-specific antigen; TRP, transient receptor potential; TRPM, TRP melastatin; TRPV, TRP vanilloid.

increase the accuracy in the subcategorization of prostate cancers and help in identifying those with an aggressive phenotype. It should be noted that the examples discussed here required the analysis of excised tumor tissue or biopsies by in situ hybridization, immunohistochemistry or similar techniques. In order to simplify the use of these biomarkers in the clinical routine, it would be a real challenge to detect aberrant expression patterns of these TRP genes in body fluids. Indeed, the progression of many tumor types is associated with the presence of circulating tumor cells in the peripheral blood of the patient.

The second question arising from the studies described here concerns the genuine feasibility of TRP proteins as drug targets. In principle, TRP channels are attractive drugs targets since they comprise extracellular domains that can bind ligands or antibodies and at the functional level, they affect key processes in prostate cancer progression such as proliferation, apoptosis and migration. Indeed drugs can affect ion channel function through several different mechanisms ranging from the classic pore block, over allosteric regulation due to (specific) drug binding to the target protein, to the allosteric regulation that arises from (more nonspecific) drug-induced changes in 
Ion channels in prostate carcinogenesis

D Gkika and N Prevarskaya

676

lipid packing adjacent to the protein and in bilayer material properties. ${ }^{32,33}$ This area of research is particularly significant, as the potentil for the pharmacological modulation of channels is one of the key advantages over other targets, which may be restricted to siRNA or gene therapy approaches. For instance, the newly identified carboyamide WS-12 is the most potent TRPM8 agonist ${ }^{34}$ and could constitote a good candidate for drug development. In addition, the development of humanized inhibitory antibodies to extracellular domains of TRPV6 and TRPV2 channels may provide the desired specificity for a successful use in vive. Finally, in vive experiments and human trials will have to define the real potentials and limitations of TRP channels as a drug targets.

1 Motel C, Birnbaumer L, Flockerzi V, Bindels RJ, Buford EA et al. A unified nomenclature for the superfamily of TRP cation channels. Mol Cell 2002; 9: 229-31.

2 Clapham DE. TRP channels as cellular sensors. Nature 2003; 426: 517-24.

3 Clapham DE, Runnels LW, Strubing C. The TRP ion channel family. Nat Rev Neurosci 2001; 2: 387-96.

4 Nilius B, Owsianik G, Vets T, Peters JA. Transient receptor potential cation channels in disease. Physio Rev 2007; 87: 165-217.

5 Duncan LM, Deeds J, Hunter J, Shan J, Holmgren LM et al. Down-regulation of the novel gene melastatin correlates with potential for melanoma metastasis. Cancer Res 1998; 58: 1515-20.

6 Thebault S, Flourakis M, Vanoverberghe K, Vandermoere F, Roudbaraki M et al. Differential role of transient receptor potential channels in $\mathrm{Ca}^{2+}$ entry and proliferation of prostate cancer epithelial cells. Cancer Res 2006; 66: 2038-47.

7 Tsavaler L, Shapero MH, Morkowski S, Laws R. Trp-p8, a novel prostate-specific gene, is up-regulated in prostate cancer and other malignancies and shares high homology with transient receptor potential calcium channel proteins. Cancer Res 2001; 61: 3760-9.

8 Wissenbach U, Niemeyer BA, Fixemer T, Schneidewind A, Trust C et al. Expression of CaT-like, a novel calcium-selective channel, correlates with the malignancy of prostate cancer. J Biol Chem 2001; 276: 19461-8.

9 Henshall SM, Afar DE, Heller J, Horvath LG, Quinn DI et al. Survival analysis of genome-wide gene expression profiles of prostate cancers identifies new prognostic targets of disease relapse. Cancer Res 2003; 63: 4196-203.

10 Bidaux G, Flourakis M, Thebault S, Zholos A, Beck B et al. Prostate cell differentiation status determines transient receptor potential melastatin member 8 channel subcellular localization and function. J Chin Invest 2007; 117: 1647-57.

11 Bidaux G, Roudbaraki M, Merle C, Crepin A, Delcourt P et al. Evidence for specific TRPM8 expression in human prostate secretory epithelial cells: functional androgen receptor requirement. Endocr Relat Cancer 2005; 12: 367-82.

12 Thebault S, Lemonnier L, Bidaux G, Flourakis M, Bavencoffe A et al. Novel role of cold/ menthol-sensitive transient receptor potential melastatine family member 8 (TRPM8) in the activation of store-operated channels in LNCaP human prostate cancer epithelial cells. J Biol Chem 2005; 280: 39423-35.

13 Zhang L, Barit GJ. Evidence that TRPM8 is an androgen-dependent $\mathrm{Ca}^{2+}$ channel required for the survival of prostate cancer cells. Cancer Res 2004; 64: 8365-73.

14 Prevarskaya N, Bhang L, Barit G. TRP channels in cancer. Biochim Biophys Acta 2007; 1772: 937-46.
15 Skryma R, Marion P, Bourhis XL, Coppenolle FV, Shuba Y et al. Store depletion and store-operated $\mathrm{Ca}^{2+}$ current in human prostate cancer LNCaP cells: involvement in apoptosis. J Physiol 2000; 527 (Pt 1): 71-83.

16 Vanden Adele F, Skryma R, Shuba Y, van Coppenolle F, Slomianny C et al. Bcl-2dependent modulation of $\mathrm{Ca}^{2+}$ homeostasis and store-operated channels in prostate cancer cells. Cancer Cell 2002; 1: 169-79.

17 Vanoverberghe K, Vanden Adele F, Mario P, Lepage G, Roudbaraki M et al. $\mathrm{Ca}^{2+}$ homeostasis and apoptotic resistance of neuroendocrine-differentiated prostate cancer cells. Cell Death Differ 2004; 11: 321-30.

18 Gkika D, Flourakis M, Lemonnier L, Prevarskaya N. PSA reduces prostate cancer cell motility by stimulating TRPM8 activity and plasma membrane expression. Oncogene 2010; 29: 4611-6.

19 Heidtmann HH, Nettelbeck DM, Mingels A, Jager R, Walker HG et al. Generation of angiostatin-like fragments from plasminogen by prostate-specific antigen. $\mathrm{Br} J$ Cancer 1999; 81: 1269-73.

20 Yang $\mathrm{ZH}$, Wang $\mathrm{XH}$, Wang HP, Mu LQ. Effects of TRPM8 on the proliferation and motility of prostate cancer PC-3 cells. Asian J Androl 2009; 11: 157-65.

21 Fixemer T, Wissenbach U, Flockerzi V, Bonkhoff $\mathrm{H}$. Expression of the $\mathrm{Ca}^{2+}$-selective cation channel TRPV6 in human prostate cancer: a novel prognostic marker for tumor progression. Oncogene 2003; 22: 7858-61.

22 Lehen'kyi V, Flourakis M, Skryma R, Prevarskaya N. TRPV6 channel controls prostate cancer cell proliferation via $\mathrm{Ca}^{2+} /$ NFAT-dependent pathways. Oncogene 2007; 26 : 7380-5.

23 Katsogiannou M, El Boustany C, Gackiere F, Delcourt P, Athias A et al. Caveolae contribute to the apoptosis resistance induced by the alpha ${ }_{1 \mathrm{~A}}$-adrenoceptor in androgen-independent prostate cancer cells. PLo One 2009; 4: e7068.

24 Monet M, Lehen'kyi V, Gackiere F, Firlej V, Vandenberghe M et al. Role of cationic channel TRPV2 in promoting prostate cancer migration and progression to androgen resistance. Cancer Res 2010; 70: 1225-35.

25 Weinstein MH, Martin AW, Veltri RW, Epstein JI. Neuroendocrine differentiation in prostate cancer: enhanced prediction of progression after radical prostatectomy. Human Pathol 1996; 27: 683-7.

26 Kanzaki M, Chang YQ, Mashima H, Li L, Shibata H et al. Translocation of a calciumpermeable cation channel induced by insulin-like growth factor-I. Nat Cell Biol 1999; 1: 165-70.

27 Monet M, Gkika D, Lehen'kyi V, Pourtier A, Vanden Adele F et al. Lysophospholipids stimulate prostate cancer cell migration via TRPV2 channel activation. Biochim Biophys Alta 2009; 1793: 528-39.

28 Hoo F, Tan M, Xu X, Han J, Miller DD et al. Lysophosphatidic acid induces prostate cancer PC 3 cell migration via activation of $\mathrm{LPA}_{1}, \mathrm{p} 42$ and p38alpha. Biochim Biophys Alta 2007; 1771: 883-92.

29 Raj GV, Sekula JA, Gro R, Madden JF, Daaka Y. Lysophosphatidic acid promotes survival of androgen-insensitive prostate cancer PC 3 cells via activation of NFkappa. Prostate 2004; 61: 105-13.

30 Daaka Y. Mitogenic action of LPA in prostate. Biochim Biophys Acta 2002; 1582 : 265-9.

31 Chang L, Barritt GJ. TRPM8 in prostate cancer cells: a potential diagnostic and prognostic marker with a secretory function? Endocr Relat Cancer 2006; 13: 27-38.

32 Andersen OS. Perspectives on how to drug an ion channel. J Gen Physio 2008; 131: 395-7.

33 Arcangeli A, Crociani O, Lastraioli E, Maxi A, Pillozzi S et al. Targeting ion channels in cancer: a novel frontier in antineoplastic therapy. Cur Med Chem 2009; 16: 66-93.

34 Beck B, Bidaux G, Bavencoffe A, Lemonnier L, Thebault S et al. Prospects for prostate cancer imaging and therapy using high-affinity TRPM8 activators. Cell Calcium 2007; 41: 285-94.

Asian Journal of Andrology 